\title{
Structure of oxygen-plasma-treated ultrathin praseodymia films on $\mathrm{Si}(111)$
}

\author{
S. Gevers, ${ }^{1}$ T. Weisemoeller, ${ }^{1}$ A. Schaefer, ${ }^{2}$ V. Zielasek, ${ }^{2}$ M. Bäumer, ${ }^{2}$ and J. Wollschläger ${ }^{1, *}$ \\ ${ }^{1}$ Fachbereich Physik, University of Osnabrück and Center of Interface Science, Barbarastrasse 7, D-49069 Osnabrück, Germany \\ ${ }^{2}$ Institute of Applied and Physical Chemistry, University of Bremen, Leobener Strasse NW2, D-28359 Bremen, Germany
}

(Received 10 December 2010; published 25 May 2011)

\begin{abstract}
Ultrathin praseodymia films, which have been oxidized by molecular oxygen, have been treated additionally with oxygen plasma to increase their oxidation state. The structure and morphology of the films have been investigated by $\mathrm{x}$-ray diffraction and $\mathrm{x}$-ray reflectometry. Thorough analysis of these measurements gives information regarding modifications of the oxide film structure (especially the vertical lattice constants) due to the oxygen content and interface silicate formation before and after oxygen plasma treatment. Large parts of the plasma-treated samples exhibit a significantly higher oxygen content compared to the untreated samples; this is attributed to the formation of stoichiometric $\mathrm{PrO}_{2}$. The remaining film has only a small oxygen deficiency. Thus, a more homogeneous film structure is formed by exposure to oxygen plasma. Furthermore, no additional silicate interface formation can be detected.
\end{abstract}

DOI: 10.1103/PhysRevB.83.193408

PACS number(s): 68.35.Fx, 68.49.Uv, 68.55.aj, 68.55.Nq

Rare earth oxides (REOs) are of interest for many applications in the fields of heterogeneous catalysis ${ }^{1}$ and microelectronics. ${ }^{2}$ For instance, REO films are of potential use to improve the performance and functionality of future semiconductor devices by integrating alternative semiconductor materials into the present $\mathrm{Si}$ technology. ${ }^{3,4}$ In this regard, single-crystalline REO films are under discussion as highly functional insulating buffer material to form so-called engineered $\mathrm{Si}$ wafers. The main research fields are integrated germanium-on-insulator systems aiming to boost the sub-45-nm complementary metal-oxide-semiconductor technologies and further to achieve the cost-effective monolithic integration of III-V optoelectronic materials ( $\mathrm{GaAs}$ ) on the $\mathrm{Si}$ wafer platform.

Praseodymia is a promising candidate for these insulating buffer materials and previous studies show that crystalline Ge films grow epitaxially on praseodymia-silicon heterostructures. ${ }^{4}$ Since as-grown praseodymia films initially have hexagonal structure (hex- $\mathrm{Pr}_{2} \mathrm{O}_{3}$ ), it is necessary to transform the structure of these films to $\mathrm{PrO}_{2}$ (fluorite structure) to obtain stacking-twin-free Ge films. ${ }^{4,5}$ For this purpose hex- $\mathrm{Pr}_{2} \mathrm{O}_{3}$ films are usually exposed to molecular oxygen at high pressure, but full transformation to $\mathrm{PrO}_{2}(111)$ has not been achieved yet. ${ }^{6-8}$ Instead, the films decompose into two laterally coexisting oxide species with different lattice parameters and significantly different stoichiometries $\left(\mathrm{PrO}_{1.833}\right.$, $\mathrm{PrO}_{2-\Delta}$ ) as shown by detailed analysis of the praseodymia Bragg peaks. This is disadvantageous for the quality of the subsequently grown Ge film due to the imperfect oxygen sublattice. The thickness of an interface layer consisting of both species increases with higher annealing temperatures, too. This interface exhibits negative effects on the dielectric properties of the oxide film. ${ }^{5}$

Schaefer et al. ${ }^{8}$ recently established an alternative technique to oxidize praseodymia films on $\mathrm{Si}(111)$ by exposure to cold oxygen plasma. Here, x-ray photoelectron spectroscopy (XPS) supported by low-energy electron diffraction (LEED) and $\mathrm{x}$-ray diffraction (XRD) demonstrated that the near-surface region of this praseodymia film has a higher oxidation state compared to the samples annealed in molecular oxygen. Our work, presented here, focuses on more detailed investigations of the structure of oxygen-plasma-treated praseodymia films by XRD and X-ray reflectometry (XRR). Particular features of the praseodymia film structure are obtained from our careful XRD analysis using kinematic diffraction theory combined with XRR analysis based on the Parratt algorithm. Thus, we determine the composition of the oxide films regarding different praseodymia phases (identified by the vertical lattice constants) and their ordering. For instance, we obtain information concerning not only the lateral phase separation but also the vertical stacking of the different phases.

Ultrathin hex- $\operatorname{Pr}_{2} \mathrm{O}_{3}$ films with $15 \mathrm{~nm}$ film thickness were deposited on clean boron-doped $\mathrm{Si}(111)$ substrates $\left(\rho=5-15 \Omega \mathrm{cm}\right.$, off oriented by $\left.0.35^{\circ} \pm 0.15^{\circ}\right) .{ }^{9}$ These samples were annealed ex situ in $1 \mathrm{~atm}$ oxygen at $450{ }^{\circ} \mathrm{C}$ for $30 \mathrm{~min}$ to obtain heteroepitaxial praseodymia films with fluorite structure $(F m \overline{3} m$ in Hermann-Mauguin notation) and exclusive $B$-type stacking. ${ }^{10}$ The oxygen plasma treatment was performed afterward for $60 \mathrm{~min}$ at 9 mbar oxygen partial pressure and a gas flow of $15 \mathrm{sccm}$ (standard cubic centimeters per minute) using an ultrahigh-vacuum-compatible, capacitively coupled rf plasma source ${ }^{11}$ with a power of $30 \mathrm{~W}$. After in situ XPS and LEED measurements, the samples were transferred under clean oxygen atmosphere to beamline BW2 at DESY (Hamburg, Germany) to determine the structure of both untreated and oxygen-plasma-treated oxide films. Here, ex situ XRD and XRR measurements were performed in $\Theta-2 \Theta$ geometry using a six-circle diffractometer at photon energy of $10 \mathrm{keV}$. A one-dimensional $\mathrm{x}$-ray photon detector provides additional lateral information via reciprocal space mapping (RSM).

The XRR measurements and calculated intensities of the untreated and the oxygen-plasma-treated samples are presented in Fig. 1. Both samples exhibit well-defined intensity oscillations (fringes), pointing to homogeneous film structures, but the fringes of the plasma-treated sample are more pronounced. Therefore, the roughness of the plasmatreated sample decreases with respect to the untreated sample, probably due to the previously reported cleaning of the film surface. ${ }^{8}$ The model which was used to calculate the intensity 


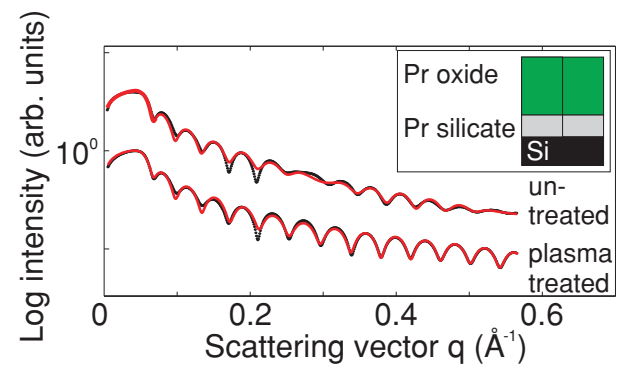

FIG. 1. (Color online) XRR data (dots) and calculated fitting curves (solid lines) of untreated and oxygen-plasma-treated samples. Inset: Model used for the XRR calculations.

distribution is also shown in Fig. 1. This model, consisting of the praseodymia film on top of a silicate layer above the Si substrate, was established in our earlier work. ${ }^{7}$ Here, the film structure was further split into two laterally separated columns to reflect decomposition of the praseodymia films obtained from our XRD analysis (see below). Both calculations according to the proposed model show very good agreement with the experimental data. The mean praseodymia film thickness of the untreated sample results in $14.7 \pm 0.5 \mathrm{~nm}$ with a mean silicate layer thickness of $1.4 \pm 0.5 \mathrm{~nm}$. The rms roughness of the untreated praseodymia surface is about $2.1 \pm 0.5 \mathrm{~nm}$. Similar values for the mean oxide film thickness $(14.9 \pm 0.5 \mathrm{~nm})$ and the silicate thickness $(1.7 \pm 0.5 \mathrm{~nm})$ are observed after plasma treatment. The rms roughness of the film surface, however, is significantly decreased to $1.1 \pm$ $0.5 \mathrm{~nm}$.

Figure 2 presents specular RSMs of the $(00 L)_{S}$ rod for the untreated (left) and the oxygen-plasma-treated (right) samples at $L \approx 1$ and $L \approx 2$, respectively. Here, $L=d_{\mathrm{Si}} Q_{\perp} / 2 \pi$ denotes the vertical scattering vector $Q_{\perp}$ in reciprocal lattice units which is scaled to the $\operatorname{Si}(111)$ layer distance $d_{\mathrm{Si}}=$ $314 \mathrm{pm}$. The axes are labeled in surface coordinates $(H K L)_{S}$ corresponding to the (111) orientation of the $\mathrm{Si}$ substrate lattice. Thus, the Bragg peaks denoted by $(00 L)_{S}$ in surface notation are equivalent to the $(L L L)_{B}$ Bragg peaks in bulk notation. In all RSMs sharp Bragg peaks are visible due to the $\mathrm{Si}(111)$ substrate (marked with triangles). Furthermore, broad Bragg peaks can be observed close to integer $L$ values which are caused by the ultrathin praseodymia film. Strong interference effects between the signals from substrate and crystalline film occur close to $L \approx 1$ so that enhanced diffraction intensity appears for the crystal truncation rod at $K=0$ (cf. streaks and fringes in Fig. 2 for the region close to $L=1$ ). This complicates the analysis of the data in this region with respect to the structure of the praseodymia film. ${ }^{12}$ For instance, the amorphous silicate interface layer, which separates crystalline parts of the praseodymia film and $\mathrm{Si}$ substrate, leads to additional oscillations (fringes) which are not observed at $L \approx 2$ where only slight interference effects between film and substrate can be observed, since the Si Bragg peak is kinematically forbidden. Due to deviations from the spherical electron distribution of the $\mathrm{Si}$ atoms, however, a small Si intensity which rapidly decreases with $L \neq 2$ can be observed here. Since the intensity of the oxide Bragg peaks is still adequate, this region is used for a detailed analysis of the diffracted intensity.The analysis of the $\Theta-2 \Theta$ scan at $L \approx 2$

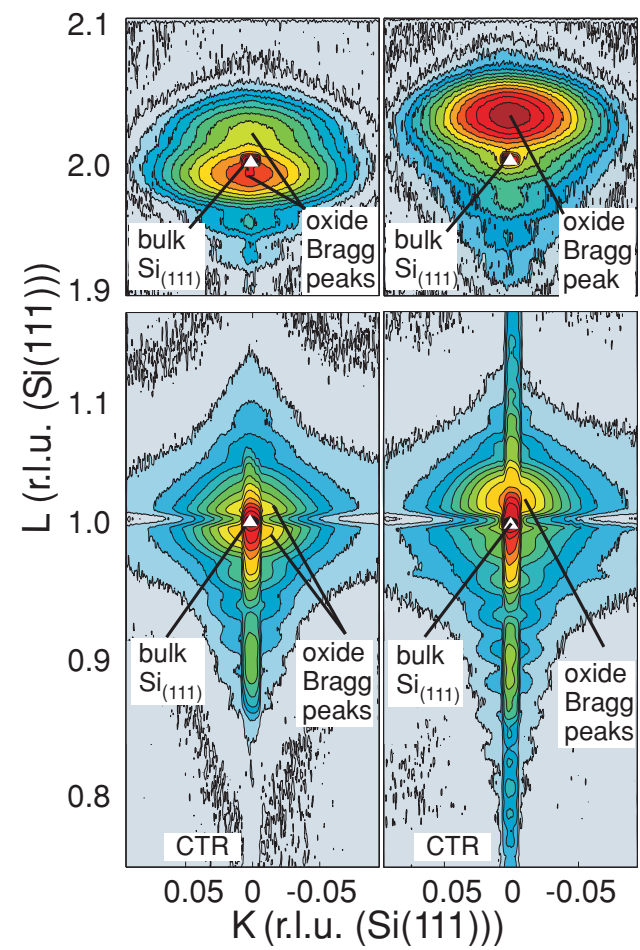

FIG. 2. (Color online) Specular reciprocal space maps of the untreated sample (left) and the oxygen-plasma-treated sample (right) at $L \approx 1$ and $L \approx 2$

for the untreated sample is shown in Fig. 3(a). At first sight, the scan exhibits two broad Bragg peaks caused by two laterally coexisting oxide species, as already reported for this kind of postdeposition-annealed samples. ${ }^{7,10}$ In the previous report the oxide Bragg peak obtained at lower $L$ values was classified as a $\mathrm{PrO}_{2-\Delta}$ phase and the other peak at higher $L$ values as a vertically strained $\mathrm{PrO}_{2}$ species. Since the integration of oxygen atoms into the praseodymia film leads to the formation of $\mathrm{Pr}^{4+}$ ions, which have a smaller ionic radius than $\operatorname{Pr}^{3+}$ ions, ${ }^{13}$ smaller lattice constants (corresponding to higher $L$ values for Bragg peaks) can indirectly be attributed to a higher oxygen content of the oxide species. For this reason, we will show below that the plasma treatment leads to even higher oxidization states than reported previously. This also suggests that the oxygen content of the untreated samples is significantly lower than the content of the plasma-treated praseodymia film as supported by $\mathrm{x}$-ray photoelectron spectroscopy for the surface region. ${ }^{8}$ Therefore, we will refer in the following to these oxide phases as $\mathrm{PrO}_{1.833}$ and $\mathrm{PrO}_{2-\Delta}$, respectively.

Based on the kinematic diffraction theory, we analyzed the diffracted intensity close to $L=2$. The main adjustable parameters in the calculations are the layer distances $d_{i}$ and the number of layers $N_{i}$ as well as the top and bottom roughnesses $\Phi_{i}^{(t)}$ and $\Phi_{i}^{(b)}$ of the particular film parts. The intensity from a single column is obtained by adding up coherently the diffracted amplitudes of all layers. The total intensity is then derived by adding up incoherently the amplitudes diffracted from each lateral coexisting column according to the random phase approximation. The sharp $\mathrm{Si}$ Bragg peak intensity, however, is always neglected since a spherical electron distribution of the $\mathrm{Si}$ atoms is assumed in 

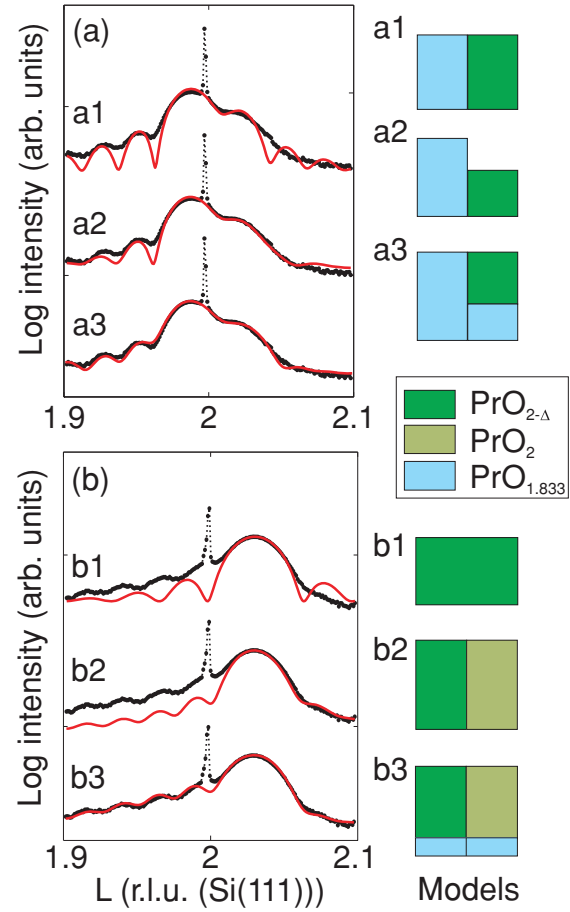

FIG. 3. (Color online) Specular $\Theta-2 \Theta$ scans of the untreated sample (a) and the oxygen-plasma-treated sample (b) at $L \approx 2$ and calculations (solid lines) with different structure models (right side).

the kinematic calculations. Furthermore, the mean absolute error of logarithmized data is used as the minimized error function. In contrast to previous reports, ${ }^{7}$ this analysis shows that the diffracted intensity cannot be modeled with only two oxide phases of similar thickness which coexist laterally [see Fig. 3(a1)]. On the one hand, the calculated Bragg peak at higher $L$ values is considerably smaller compared to the experimental data and, on the other hand, the fringes due to the film thickness are poorly described. A better matching between calculation and measurement can be achieved by decreasing the layer thickness of the $\mathrm{PrO}_{2-\Delta}$ oxide species [see Fig. 3(a2)] so that both Bragg peaks are reproduced in very good agreement with the measurement. However, the fringes are still poorly described and a disagreement between the resulting film thicknesses $\left(D_{\mathrm{PrO}_{1.833}}=12.3 \mathrm{~nm}\right.$ and $D_{\mathrm{PrO}_{2-\Delta}}=$ $6.9 \mathrm{~nm})$ and the total film thickness determined by XRR measurements $(14.7 \mathrm{~nm})$ is also obtained. Because the XRR measurements suggest an interface layer with $1.5 \mathrm{~nm}$ thickness for both laterally separated phases, the reduced $\mathrm{PrO}_{2-\Delta}$ film thickness cannot be explained by an increased interface layer. Therefore, we suggest that $\mathrm{PrO}_{1.833}$ has been formed below the
$\mathrm{PrO}_{2-\Delta}$ phase. The further iteration of our calculation based on this model includes a homogeneous $\mathrm{PrO}_{1.833}$ column and an additional column of vertically stacked $\mathrm{PrO}_{2-\Delta}$ and $\mathrm{PrO}_{1.833}$. The model shows good agreement between calculated and measured XRD data [see Fig. 3(a3); film thicknesses are presented in Table I]. Our XRD analysis is also supported by XRR results, supposing that a small amorphous hydroxide layer has formed at the film surface due to exposure to ambient conditions during the ex situ measurements. The different oxide species, however, cannot be distinguished with XRR since they exhibit almost identical electron densities (see Fig 1). The vertical lattice constants of the $\mathrm{PrO}_{1.833}$ species are $d_{1, \mathrm{PrO}_{1.833}}=315 \mathrm{pm}$ and $d_{2, \mathrm{PrO}_{1.833}}=317 \mathrm{pm}$ for both columns, while $\mathrm{PrO}_{2-\Delta}$ has a vertical lattice constant of $311 \mathrm{pm}$. If lateral pinning of the oxide phases is considered (see below), an understoichiometry of $\Delta=0.06 \pm 0.02$ is estimated from the vertical lattice constants (see Table I).

The intensity analysis at $L=2$, however, provides no information about the stacking order of the praseodymia species within the second column. In-plane grazing incidence $\mathrm{XRD}$ proves that the entire oxide film is pinned laterally to the lattice constant of hex- $\operatorname{Pr}_{2} \mathrm{O}_{3}\left(a_{\|}=386 \mathrm{pm}\right){ }^{6}$ which is considerable closer to the lattice constant of $\mathrm{PrO}_{1.833}$ than that of $\mathrm{PrO}_{2}$. Therefore, lateral strain effects increase close to the interface if more oxygen is incorporated into the film as is necessary for praseodymia with $\mathrm{PrO}_{1.833}$ stoichiometry. Furthermore, it is reasonable to assume that $\mathrm{PrO}_{1.833}$ with small oxygen content is formed close to the interface and not at the top of this oxide column since the strain is minimized in this case. The lateral size of the crystallites were calculated from the full width at half maximum of the oxide Bragg peaks [see Fig. 2(a)]. Here, the homogeneous and the inhomogeneous columns exhibit sizes of $90 \pm 10$ and $30 \pm 10 \mathrm{~nm}$, respectively.

After oxygen plasma treatment, the $\Theta-2 \Theta$ scan of the sample does not exhibit an oxide Bragg peak at $L \leqslant 2$ [see Fig. 3(b1)]. Thus, the $\operatorname{PrO}_{1.833}$ part of the oxide film is oxidized further and vanishes with plasma treatment. The position of the oxide Bragg peak for $L \geqslant 2$ also shows that its lattice constant is decreased, as expected for praseodymia of higher oxidation state. ${ }^{13}$ Careful analysis of the data, however, shows that models with only one oxide species result in poor agreement with the experimental data and that the obtained film thickness of $7.5 \pm 0.5 \mathrm{~nm}$ is significantly smaller than determined by XRR. An altered model of the diffracted intensity using two laterally coexisting oxide species with slightly different layer distances $d$ exhibits a significantly better agreement with the diffraction intensity, in particular regarding the fringe positions at lower $L$ values [see Fig. 3(b2)]. Nevertheless, a considerable lack of intensity is observed here, too.

TABLE I. Oxide film thicknesses $D$ and layer distances $d$ obtained from the XRD measurements. The PrO ${ }_{2}$ species are labeled with (*).

\begin{tabular}{|c|c|c|c|c|c|}
\hline \multirow[b]{2}{*}{ Material } & & \multicolumn{2}{|c|}{ Untreated } & \multicolumn{2}{|c|}{ Plasma treated } \\
\hline & & $1 \mathrm{col}$. & $2 \mathrm{col}$. & $1 \mathrm{col}$. & $2 \mathrm{col}$. \\
\hline \multirow[t]{2}{*}{$\mathrm{PrO}_{2-\Delta} / \mathrm{PrO}_{2}(*)$} & $D( \pm 0.1)$ & - & $7.8 \mathrm{~nm}$ & $12.4 \mathrm{~nm}$ & $12.6 \mathrm{~nm}(*)$ \\
\hline & $d( \pm 1)$ & - & $311 \mathrm{pm}$ & 309 pm & $307 \mathrm{pm}(*)$ \\
\hline \multirow[t]{2}{*}{$\mathrm{PrO}_{1.833}$} & $D( \pm 0.1)$ & $13.2 \mathrm{~nm}$ & $5.7 \mathrm{~nm}$ & $1.6 \mathrm{~nm}$ & $1.6 \mathrm{~nm}$ \\
\hline & $d( \pm 1)$ & $315 \mathrm{pm}$ & $317 \mathrm{pm}$ & $315 \mathrm{pm}$ & $315 \mathrm{pm}$ \\
\hline
\end{tabular}


Finally, we assumed two laterally coexisting oxide phases with $\mathrm{PrO}_{2}$ and $\mathrm{PrO}_{2-\Delta}$ stoichiometry which exhibit very similar vertical lattice constants and added two small $\mathrm{PrO}_{1.83}$ fractions below these main oxide parts. Here, the measured intensity distribution is in good agreement with the calculation [see Fig. 3(b3)]. The resulting overall film thickness of $14.1 \pm 0.1 \mathrm{~nm}$ is also in good agreement with the XRR analysis (see Table I). A slight increase of the crystalline oxide layer thickness compared to the untreated sample also suggests a cleaning process of the film surface since the layer contaminated by hydroxide due to exposure to ambient conditions is significantly smaller. The vertical layer constants of both top oxide species are significantly decreased compared to that of the $\mathrm{PrO}_{2-\Delta}$ species of the untreated sample (see Table I) and even smaller than the bulk $\mathrm{PrO}_{2}$ value. This effect can be attributed to the tetragonal distortion of the film caused by the lateral pinning at the interface. ${ }^{6}$ The oxide species with larger lattice constant, however, still exhibits oxygen vacancies, while the second species with smaller lattice constant is more oxidized. Thus, the species are identified as $\mathrm{PrO}_{2-\Delta}$ and $\mathrm{PrO}_{2}$ according to previous XPS studies. ${ }^{8}$

Both smaller parts at the bottoms of the columns obtained from our complete analysis [see Fig. 3(b3)] have a vertical lattice constant of $315 \mathrm{pm}$ and can be identified with the $\mathrm{PrO}_{1.833}$ species reported above for the untreated sample. Since we demonstrated before [see Fig. 3(a3)] that one column of the untreated sample is completely $\mathrm{PrO}_{2-\Delta}$, we assume that the plasma treatment transforms the first $12 \mathrm{~nm}$ of the crystalline oxide film while the region close to the interface is partly reduced due to the increased sample temperature during plasma treatment and the influence of the silicate interface layer. Regarding the crystallite sizes, only one mean value of
$115 \pm 10 \mathrm{~nm}$ could be observed for both columns because of the slight separation of the oxide Bragg peaks. Nevertheless, it seems that the lateral film structure is more homogeneous after plasma treatment. Furthermore, the XRD analysis close to $L \approx 1$ shows no significant changes of the fringe positions before and after plasma treatment (see Fig. 1). Thus, the interface thickness of the amorphous silicate interface is not changed by the plasma treatment, in agreement with the XRR results.

In summary, the structural analysis of oxygen-plasmatreated films, using detailed XRD calculations within the kinematic diffraction theory, verified that oxygen plasma treatment of ultrathin preoxidized praseodymia films enhances the oxygen content significantly without increasing the silicate interface between oxide and substrate. Plasma-activated atomic oxygen occupies the oxygen vacancies of both phases $\mathrm{PrO}_{2-\Delta}$ and $\mathrm{PrO}_{1.833}$ so that the praseodymia film has complete $\mathrm{PrO}_{2}$ stoichiometry. The XRD analysis showed further that a small part $(<11 \%)$ of the praseodymia film still exhibits $\mathrm{PrO}_{1.833}$ stoichiometry. Nevertheless, this technique is very promising for obtaining high-quality praseodymia films with homogeneous fluorite structure of better quality than obtained up to now with other methods.

The authors acknowledge the Deutsche Forschungsgemeinschaft (DFG) via Graduate College 695 and Grant No. WO533/16 for financial support. Furthermore, D. Novikov is acknowledged for beamline support for synchrotron experiments carried out at HASYLAB/DESY. DESY is a member of the Helmholtz Association (HGF). The samples were kindly provided by T. Schroeder and A. Guissani at the IHP GmbH, Frankfurt (Oder). *joachim.wollschlaeger@uos.de

${ }^{1}$ S. K. Bhaskaran and V. T. Bhat, React. Kinet. Catal. Lett. 75, 239 (2002).

${ }^{2}$ S. W. Bedell, A. Reznicek, K. Fogel, J. Ott, and D. K. Sadana, Mater. Sci. Semicond. Process. 9, 423 (2006).

${ }^{3}$ H. Tanoto, S. F. Yoon, W. K. Loke, E. A. Fitzgerald, C. Dohrman, B. Narayanan, M. T. Doan, and C.H. Tung, J. Vac. Sci. Technol. B 24, 152 (2006).

${ }^{4}$ A. Giussani, O. Seifarth, P. Rodenbach H.-J Müssing, P. Zaumseil, T. Weisemoeller, C. Deiter, J. Wollschläger, P. Storck, T. Schroeder J. Appl. Phys. 103, 084110 (2008).

${ }^{5}$ O. Seifarth, Ch. Walczyk, G. Lupina, J. Dabrowski, P. Zaumseil, G. Weidner, H.-J. Müssig, and T. Schroeder, J. Appl. Phys. 106, 104105 (2009).

${ }^{6}$ T. Weisemoeller, F. Bertram, S. Gevers, A. Greuling, C. Deiter, H. Tobergte,M. Neumann, A. Giussani, T. Schroeder, and J. Wollschaläger, J. Appl. Phys. 105, 124108 (2009).
${ }^{7}$ S. Gevers, T. Weisemoeller, B. Zimmermann, F. Bertram, C. Deiter, and J. Wollschläger, J. Phys.: Condens. Matter 21, 441 (2009).

${ }^{8}$ A. Schaefer, S. Gevers, V. Zielasek, T. Schroeder, J. Falta, J. Wollschläger, and M. Bäumer, J. Chem. Phys. 134, 054701 (2011).

${ }^{9}$ T. Schroeder, T.-L. Lee, L. Libralesso, I. Joumard, and J. Zegenhagen, J. Appl. Phys. 97, 074906 (2005).

${ }^{10}$ T. Weisemoeller C. Deiter, F. Bertram, S. Gevers, A. Giussani, P. Zaumseil, T. Schroeder, and J. Wollschläger, Appl. Phys. Lett. 93, 032905 (2008).

${ }^{11}$ B. Gehl, U. Leist, V. Aleksandrovic, P. Nickut, V. Zielasek, H. Weller, K. Al-Shamery, and M. Bäumer, Rev. Sci. Instrum. 77, 083902 (2006).

${ }^{12}$ T. Weisemoeller, F. Bertram, S. Gevers, C. Deiter, A. Greuling, and J. Wollschläger, Phys. Rev. B 79, 245422 (2009).

${ }^{13}$ L. Eyring and N. C. Baenziger, J. Appl. Phys. 33, 428 (1962). 